\title{
Niveles de ansiedad en futbolistas de categorías inferiores
}

\section{Levels of anxiety in players of the lower categories}

\author{
Manuel Castro Sánchez ${ }^{1 *}$, Félix Zurita Ortega ${ }^{2}$, Ramón Chacón Cuberos ${ }^{3}$, Rosario Padial Ruz ${ }^{2}$ \\ y Asunción Martínez Martínez ${ }^{4}$ \\ 1 Profesor Doctor del Departamento de Educación Física de la Universidad Internacional de La Rioja (España). \\ 2 Profesorla Doctor del Área de Corporal de la Universidad de Granada (España). \\ 3 Profesor del Área de Corporal de la Universidad de Huelva (España). \\ 4 Profesora del Departamento de Métodos de Investigación y Diagnóstico en Evaluación de la Universidad de Granada (España).
}

\begin{abstract}
Resumen: Debido a la relación existente entre las variables psicológicas y el rendimiento deportivo, la psicología del deporte ha cobrado una especial relevancia en las últimas décadas, por lo que la presente investigación tiene como objetivo analizar los niveles de ansiedad en futbolistas de categorías inferiores, utilizando un diseño descriptivo de corte transversal sobre una muestra de 156 futbolistas adolescentes. Para la recogida de datos se ha utilizado un cuestionario ad-hoc para el registro de las variables sociodemográficas y el Stai-Rasgo para medir los niveles de ansiedad. Los resultados de la presente investigación indican que seis de cada diez futbolistas muestran niveles medios de ansiedad-estado y tres de cada diez alta, en cuanto a la ansiedad-rasgo, la mitad tienen una ansiedad alta y la otra mitad media. Como principal conclusión de esta investigación se extrae que el nivel de estudios de los futbolistas se relaciona con los niveles de ansiedad.

Palabras clave: Ansiedad estado; ansiedad rasgo; fútbol; adolescentes.
\end{abstract}

\section{Introducción}

El fútbol es uno de los deportes más complejos, debido a la interacción entre factores técnico-tácticos, psicológicos, afectivos y sociales (Davids, Araújo y Schuttleworth, 2005), por lo que se considera uno de los deportes más dinámicos de la actualidad (Pazo, Sáenz-López y Fradua, 2012). Debido a estas características, el fútbol es uno de los deportes con mayor atractivo y afición en Europa y América del Sur, siendo el evento deportivo más mediático y con mayor número de seguidores. En este deporte se combinan una serie de movimientos sencillos que provocan emociones en los agentes involucrados en esta modalidad deportiva (Fábregas, 2001).

Este deporte se clasifica como un deporte colectivo de oposición que confronta dos equipos formados por once jugadores cada uno, se desarrolla en un espacio claramente delimitado, consistiendo en un constante forcejeo para conseguir la posesión del balón, cuya finalidad es introducirlo en la portería contraria, evitando que entre en su portería (Morales y Guzmán, 2000).

Los factores psicológicos han cobrado una vital importancia en el ámbito deportivo desde hace décadas, siendo hoy

\footnotetext{
Dirección para correspondencia [Correspodence address]: Manuel Castro Sánchez. Departamento de Educación Física de la Universidad Internacional de La Rioja (Espańa).Av. de la Paz, 137, 26004 Logroño, La Rioja (España).E-mail: manuel.castro@unir.net
}

Abstract: Due to the relationship between psychological variables and sports performance, sports psychology has gained special relevance in recent decades, so the present research aims to analyze the levels of anxiety in players of lower categories, using a Descriptive cross-sectional design on a sample of 156 teenage soccer players. For the collection of data, an ad-hoc questionnaire was used to record the sociodemographic variables and the Stai-Trait to measure anxiety levels. The results of this research indicate that six out of ten players show average levels of anxiety-state and three out of ten high, in terms of trait-anxiety, half have high anxiety and the other half. The main conclusion of this research is that the level of studies of footballers is related to levels of anxiety.

Keywords: Anxiety state; trait anxiety; soccer; teenagers.

fundamentales en cualquier deporte de alto rendimiento, habiéndose comprobado su importancia en los resultados deportivos, por lo que en la actualidad el papel del psicólogo deportivo es fundamental en cualquier equipo deportivo que busque maximizar sus resultados. La psicología del deporte ha estudiado los procesos de ansiedad en el ámbito de la actividad física y el deporte, centrándose sobre todo en el alto rendimiento, por ser el nivel en el que se pueden encontrar niveles de ansiedad más altos (Olmedilla, Bazaco, Ortega y Boladeras, 2011).

En el contexto deportivo Weinberg y Gould (1996) definió la ansiedad como un estado psicoemocional negativo caracterizado por sensaciones en las que predomina la preocupación y el nerviosismo, relacionándose directamente con el arousal, encontrando un componente cognitivo y otro somático.

El arousal se entiende como el estado óptimo de rendimiento que puede alcanzar un individuo, ligado a sensaciones de calma, relajación y soltura (Loerhr, 1990). En este estado de alerta el deportista goza de una sensación de autocontrol y autoconfianza óptima, muy relacionado con el concepto de flow, referido a una sensación de fluir en el que el sujeto se aísla de todo centrándose plenamente en la actividad que va a desarrollar, consiguiendo enfocar todas sus habilidades, capacidades, pensamientos y emociones al desarrollo de dicha tarea (Álvarez, López, Gómez, Brito y González, 2017). 
En el contexto deportivo se ha investigado la relación entre las lesiones deportivas y los niveles de ansiedad de los deportistas, por su estrecha relación (Rosenthal, Rainey, Tognoni y Worms, 2012), encontrando que los sujetos con niveles de ansiedad elevados son más propensos a lesionarse debido al aumento del estrés que se da en situaciones estresantes como las competiciones (Yuill, Pajackkowsky, Jason y Howitt, 2012). Weinberg y Gould (2010) señalan la importancia de los factores psicológicos en el contexto deportivo en relación con la aparición de lesiones, cuando las situaciones estresantes son muy intensas y frecuentes, la posibilidad de lesión es mayor.

Existen multitud de estudios que indican la relación inversa que existe entre la práctica deportiva y los niveles de ansiedad de los sujetos (McAuley, Márquez, Jerome, Blissmer y Katula, 2002), encontrando que la ansiedad disminuye sobremanera cuando aumenta la práctica de actividad física recreativa (Castro et al., 2015; Chacón et al., 2016; Espejo et al., 2017; Olmedilla, Prieto y Blas, 2011).

Uno de los factores que se han analizado ampliamente en la psicología del deporte ha sido la relación entre la ansiedad y los estilos de enseñanza de los entrenadores, encontrando que la comunicación del entrenador con sus deportistas puede modificar los niveles de ansiedad (Smith, Smoll y Cumming, 2007).

Además se encuentran investigaciones que analizan el control de la ansiedad en contextos competitivos y de entrenamiento, encontrando que deportistas con bajos niveles de ansiedad consiguen mejores resultados en las competiciones y los sujetos con elevados niveles de ansiedad obtienen peores resultados (Fernández, Fidalgo, Zurita, García y Sánchez, 2009).

La presente investigación pretende complementar los estudios realizados en el ámbito deportivo, realizados desde diversas perspectivas, en los que se ha estudiado la relación entre los niveles de ansiedad y las lesiones deportivas (Fernández, Zurita, Linares, Ambrós, Pradas y Linares, 2014; GarcíaMas, Pujals, Fuster, Núńez y Rubio, 2014; Liberal, Escudero, Cantallops y Ponseti, 2014), la ansiedad y el liderazgo (Leal, 2016; Valdivia-Moral, 2016; Zurita, Pérez, González, Castro, Chacón y Ambrís, 2016), o relacionando los niveles de ansiedad en el ámbito del rendimiento deportivo (León-Prados, Fuentes y Calvo, 2016; Molina, Sandín y Chorot, 2014; Mora, Sousa y Cruz, 2014).

En el ámbito del fútbol se ha analizado la ansiedad con respecto a diversas variables psicológicas (Campos, ValdiviaMoral, Zagalaz, Ortega y Romero, 2017; González-Campos, 2016; Moreno-Pellicer, 2017). El presente estudio pretende investigar sobre los niveles de ansiedad de los futbolistas en relación con diversas variables sociodemográficas y deportivas, considerando estos factores cómo condicionantes de la actividad deportiva a analizar. Se considera el estudio de los niveles de ansiedad por ser un factor influyente en el desarro- llo deportivo que suele afectar al rendimiento, determinando su desempeńo. Esta investigación pretende complementar diversos estudios realizados en el ámbito deportivo en otras disciplinas como el judo o en el mismo deporte. Por lo tanto, para este estudio se ha planteado como objetivo analizar los niveles de ansiedad de futbolistas y comprobar las relaciones existentes con variables sociodemográficas y físico-deportivas.

\section{Metodología}

\section{Diseño y participantes}

En la presente investigación se realizó un estudio descriptivo de corte transversal en el que se pretende analizar la posible dependencia entre las variables sociodemográficas analizadas y el clima motivacional.

La muestra estaba compuesta por 178 futbolistas juveniles (16-18 ańos) pertenecientes al sexo masculino y federados de la provincia de Jaén, compitiendo en las categorías: División de Honor, Nacional, Preferente, Primera Provincial y Segunda Provincial. Para la elección de la muestra se realizó un muestreo de conveniencia atendiendo a las divisiones descritas. Se eliminaron 24 cuestionarios por encontrarse incompletos o mal cumplimentados. Por lo que la muestra final sobre la que se realizó la investigación se compone de 154 jugadores de fútbol federados de entre 16 y 18 años de edad $(\mathrm{M}=16,96: \mathrm{DT}=0,773)$.

\section{Variables e instrumentos}

Los instrumentos utilizados en el estudio han sido:

Ansiedad Estado-Rasgo (STAI-RASGO - State-TraitAnxietyInventory). Creada por Spielberg, Gorsuch y Lushene (1970). Se valora mediante una escala Likert, que normalmente van de uno a cinco, pero en este caso únicamente abarca cuatro opciones ( $1=$ Muy en Desacuerdo; $2=$ En Desacuerdo; $3=$ En Acuerdo y 4=Muy de Acuerdo). Formado por 40 ítems, este instrumento se ha empleado en el ámbito deportivo (Johnson eIvarsson, 2011) presentando un coeficiente de fiabilidad alpha de Cronbach $(\alpha=.930)$ para los ítems que miden ansiedad/estado y $\alpha=.900$ para los ítems ansiedad/ rasgo (Spielberger, Gorsuch, Luschene, Vagg, yJacobs, 1983). En este trabajo presenta respectivamente un alpha $\alpha=.783 \mathrm{y}$ $\alpha=.732$.

Se ha utilizado este instrumento para medir la ansiedad por su amplio uso por parte de la comunidad científica en contextos deportivos, utilizado en estudios como el de Valdivia-Moral, Zafra, Zurita, Castro-Sánchez, Muros y CofreBolados (2016) o el de Zafra-Santos (2016).

Cuestionario Ad Hoc, Se utilizó un cuestionario de elaboración propia para el registro de variables de tipo sociodemográfico y aquellas relacionadas con la práctica deportiva 
(Estudios, Trabajo, Demarcación, División y Años de práctica federada).

\section{Procedimiento}

Se contactó con los presidentes de los equipos para informarles sobre la naturaleza del estudio explicando los objetivos del estudio y solicitando su colaboración, informando de lo que se pretendía realizar en el estudio. Se concertaron visitas con los clubs para realizar la toma de datos, en la que siempre se contó con la presencia del investigador con el fin de resolver dudas y asegurar una correcta cumplimentación de los cuestionarios.

El proceso de recogida de datos tuvo una duración de un mes y medio, transcurriendo entre los meses de Marzo y Abril de 2017.

La presente investigación ha respetado el derecho de confidencialidad de los participantes, siguiendo las normas éticas del Comité de Investigación y Declaración de Helsinki de 1975. Se contó en todos los casos con el consentimiento informado de los padres o tutores legales, respetando siempre el resguardo a la confidencialidad.

\section{Análisis de los datos}

El análisis estadístico de los datos se realizó mediante el software IBM SPSS en su versión 23.0. Se ha comprobado la distribución de la normalidad de la muestra con las pruebas de Kolmogorov-Smirnov y Saphiro-Wilk, observándose que los valores seguían una tendencia normal, por lo que se utilizaron pruebas paramétricas. Los descriptivos básicos se definieron a través de frecuencias y medias. Para ver las diferencias existentes entre las variables se utilizó ANOVA de un factor, aplicando la prueba de Bonferroni a posteriori para comprobar las diferencias inter-grupo.

Se realizaron tablas de contingencia, aplicando el test de Chi cuadrado para evaluar si existían diferencias en el número de casos de ansiedad estado (baja, media y alta) y ansiedad de rasgo (baja, media y alta) en función de las variables de agrupación (categoría, edad, demarcación, nivel de estudios, años federado y trabajo). Sin embargo, al observar que el número de casos que presentaban ansiedad de estado y rasgo baja era siempre muy reducido (siempre menos de 5 casos), se optó por eliminar esta variable del análisis y realizar una comparación entre ansiedad de estado media y alta, y ansiedad de rasgo media y alta.

Lo mismo ocurrió al aplicar el test de Chi cuadrado a las diferencias en el número de casos de ansiedad estado (baja, media y alta) y ansiedad de rasgo (baja, media y alta) en función de los años que llevaban los jugadores federados, observando que el número de casos de tiempo federados, la opción "Menos de un año" y "Entre uno y dos años" consta- ban de cuatro casos cada uno, por lo que se decidió eliminar las variables, y realizar una comparación entre los niveles de ansiedad y los deportistas que llevaban federados "Entre dos y tres años" y los que llevaban "Más de cinco años federados".

\section{Resultados}

En la presente investigación, que ha contado con una muestra de 156 futbolistas, se ha determinado que el extracto de edad con mayor representación tenía 17 años $(40,4 \%$; n=63), seguido con los sujetos que tenían 16 ańos $(32,1 \%$; $=50)$, únicamente hubo un $27,6 \%(n=43)$ de participantes de 18 ańos de edad.

Respecto a la categoría en la que jugaban los futbolistas, el estudio ha contado con un 26,3\% $(n=41)$ de jugadores que competían en División de Honor, seguido del 22,4\% ( $\mathrm{n}=35)$ que lo hacían en Primera Provincial, el 19,9\% ( $\mathrm{n}=31$ ) lo hacían en Segunda Provincial, seguido del 17,9\% ( $\mathrm{n}=28)$ que jugaban en Nacional, y finalmente, el 13,5\% ( $n=21)$ pertenecían a la categoría Preferente.

Atendiendo a la demarcación de los futbolistas encuestados, el 36,5\% ( $n=57)$ eran defensas, 25,0\% ( $n=39)$ centrocampistas, un $21,2 \%(n=33)$ jugaban como delanteros, y únicamente el $17,3 \%(n=27)$ eran porteros.

Cuando se analizan los años que llevan federados en el fútbol los jugadores de la muestra, se comprueba como la mayoría (85,9\%; n=134) lleva más de cinco años jugando de forma federada, seguido del 9,0\% ( $n=14)$ que lleva entre tres y cuatro ańos, y únicamente se ha encontrado un 2,6\% $(n=4)$ que lleva federado entre uno y dos años, cifras idénticas a los jugadores que llevan federados menos de un año.

Se ha comprobado como la mayoría de los futbolistas analizados $(94,2 \% ; n=147)$ no trabajan, mientras que únicamente el 5,8\% ( $n=9)$ se encuentran trabajando en la actualidad.

Respecto al nivel de estudios de los deportistas que conforman el estudio, se ha encontrado que casi la mitad de la muestra $(47,4 \%$; $n=74)$ tienen estudios de Bachillerato, seguido del $32,1 \%(n=50)$ que únicamente tienen estudios de Educación Secundaria Obligatoria, el 12,8\% ( $n=20)$ se encuentran cursando Formación Profesional, y únicamente el $7,7 \%(n=12)$ están cursando estudios universitarios.

Cuando se analizan los niveles de ansiedad-estado, se encuentra que más de la mitad de los futbolistas $(61,5 \% ; n=96)$ tienen una ansiedad-estado media, seguido del 35,9\% ( $n=56)$ que tienen una ansiedad-estado alta, y únicamente el 2,6\% $(\mathrm{n}=4)$ tiene una ansiedad-estado baja. Respecto a la ansiedadrasgo, la mitad de los deportistas analizados $(50,0 \% ; \mathrm{n}=78)$ tienen una ansiedad-rasgo alta, seguido del 48,7\% ( $n=76)$ con una ansiedad-rasgo media, y únicamente el 1,3\% ( $\mathrm{n}=2)$ tienen una ansiedad-rasgo baja.

Todas las características socio-demográficas, deportivas y de niveles de ansiedad de la muestra anteriormente mencionadas, se encuentran resumidas en la Tabla 1. 
Tabla 1. Descriptivos de la muestra.

\begin{tabular}{lcc}
\hline \multirow{3}{*}{ Edad } & 16 & $32,1 \%(\mathrm{n}=50)$ \\
\cline { 2 - 3 } & 17 & $40,4 \%(\mathrm{n}=63)$ \\
\cline { 2 - 3 } Categoría & División de Honor & $26,3 \%(\mathrm{n}=41)$ \\
\cline { 2 - 3 } & Nacional & $17,9 \%(\mathrm{n}=28)$ \\
\cline { 2 - 3 } & Preferente & $13,5 \%(\mathrm{n}=21)$ \\
\cline { 2 - 3 } & Primera provincial & $22,4 \%(\mathrm{n}=35)$ \\
\cline { 2 - 3 } Demarcación & Segunda provincial & $19,9 \%(\mathrm{n}=31)$ \\
\cline { 2 - 3 } & Portero & $17,3 \%(\mathrm{n}=27)$ \\
\cline { 2 - 3 } & Defensa & $36,5 \%(\mathrm{n}=57)$ \\
\cline { 2 - 3 } & Medio & $25,0 \%(\mathrm{n}=39)$ \\
\hline \multirow{3}{*}{ Años federado } & Primer año & $2,2 \%(\mathrm{n}=33)$ \\
\cline { 2 - 3 } & Entre 1 y 2 años & $2,6 \%(\mathrm{n}=4)$ \\
\cline { 2 - 3 } & Entre 3 y 4 años & $9,0 \%(\mathrm{n}=14)$ \\
\cline { 2 - 3 } & Más de 5 años & $85,9 \%(\mathrm{n}=134)$ \\
\hline
\end{tabular}

\begin{tabular}{lcc}
\hline \multirow{2}{*}{ Trabajo } & No & $94,2 \%(\mathrm{n}=147)$ \\
\cline { 2 - 3 } & $\mathrm{Si}$ & $5,8 \%(\mathrm{n}=9)$ \\
\hline \multirow{3}{*}{ Nivel de estudios } & ESO & $32,1 \%(\mathrm{n}=50)$ \\
\cline { 2 - 3 } & Bachillerato & $47,4 \%(\mathrm{n}=74)$ \\
\cline { 2 - 3 } & Formación Profesional & $12,8 \%(\mathrm{n}=20)$ \\
\cline { 2 - 3 } Ansiedad-Estado & Universidad & $7,7 \%(\mathrm{n}=12)$ \\
\cline { 2 - 3 } & Baja & $2,6 \%(\mathrm{n}=4)$ \\
\cline { 2 - 3 } & Media & $61,5 \%(\mathrm{n}=96)$ \\
\hline \multirow{3}{*}{ Ansiedad-Rasgo } & Alta & $35,9 \%(\mathrm{n}=56)$ \\
\cline { 2 - 3 } & Baja & $1,3 \%(\mathrm{n}=2)$ \\
\cline { 2 - 3 } & Media & $48,7 \%(\mathrm{n}=76)$ \\
\hline
\end{tabular}

$\mathrm{Al}$ analizar los niveles de ansiedad de los futbolistas en función de la categoría en la que compiten (Tabla 2), no se han encontrado diferencias estadísticas $(p=.946)$ en la ansiedadestado; tampoco se han encontrado diferencias en la ansiedad-rasgo en función de la categoría $(p=.095)$.

Tabla 2. Ansiedad según categoría.

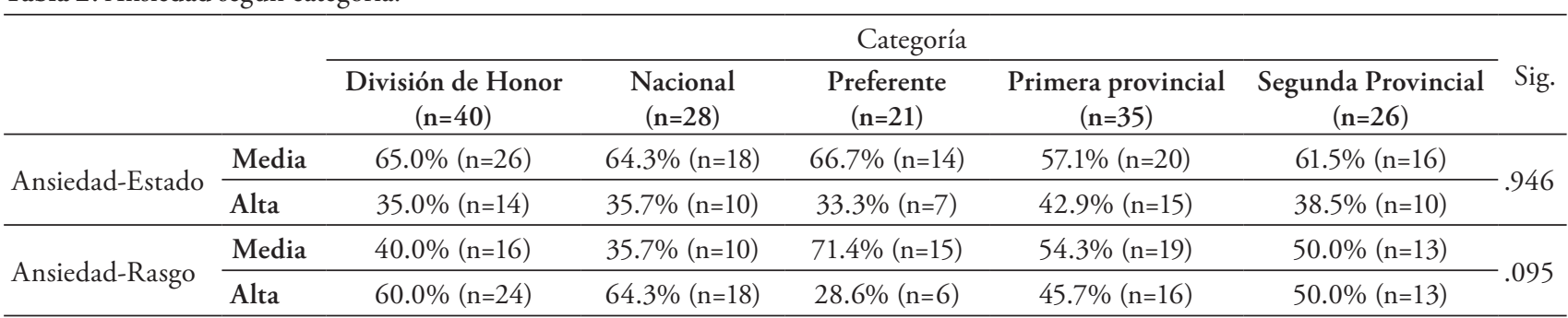

En la Tabla 3, en la que se analiza la relación entre los niveles de ansiedad-rasgo de los deportistas y su edad no se han di- ferencias estadísticas ( $p=.312)$. Tampoco se han encontrado entre la edad y la ansiedad-estado $(p=.942)$.

Tabla 3. Ansiedad según edad.

\begin{tabular}{llccc}
\hline & & \multicolumn{1}{c}{ Edad } & \\
\cline { 2 - 5 } & & $\begin{array}{c}16 \\
(\mathbf{n}=46)\end{array}$ & $\begin{array}{c}17 \\
(\mathbf{n}=61)\end{array}$ & $\begin{array}{c}18 \\
(\mathbf{n}=43)\end{array}$ \\
\hline \multirow{2}{*}{ Ansiedad-Estado } & Media & $54.3 \%(\mathrm{n}=25)$ & $63.9 \%(\mathrm{n}=39)$ & $69.8 \%(\mathrm{n}=30)$ \\
\cline { 2 - 5 } & Alta & $47.5 \%(\mathrm{n}=21)$ & $36.1 \%(\mathrm{n}=22)$ & $30.3 \%(\mathrm{n}=13)$ \\
\hline \multirow{2}{*}{ Ansiedad-Rasgo } & Media & $50.0 \%(\mathrm{n}=23)$ & $49.2 \%(\mathrm{n}=30)$ & $46.5 \%(\mathrm{n}=20)$ \\
\cline { 2 - 5 } & Alta & $50.0 \%(\mathrm{n}=23)$ & $50.8 \%(\mathrm{n}=31)$ & $53.5 \%(\mathrm{n}=23)$ \\
\hline
\end{tabular}

En la Tabla 4, en la que se analizan las diferencias entre los niveles de ansiedad-rasgo y la demarcación no se han encontrado diferencias estadísticamente significativas ( $p=.677$ ). Tampoco se observaron diferencias para la ansiedad-estado $(p=.744)$.

En la Tabla 4, que muestra las diferencias entre los niveles de ansiedad según el nivel de estudios de los deportistas, no se han encontrado diferencias estadísticamente significativas en la ansiedad-rasgo $(p=.886)$. Sin embargo, en la ansiedadestado si existen diferencias estadísticas $(p=<.05)$, encontrando que los deportistas que cursan formación profesional son los que tienen una ansiedad-estado menor, en su mayoría 88.9\% ( $\mathrm{n}=16)$ tienen una ansiedad-estado media, mientras que únicamente el $1.1 \%(\mathrm{n}=2)$ tienen ansiedad-estado alta. 
Los futbolistas que estudian bachillerato presentan los niveles de ansiedad más altos, encontrando que el $47.9 \% \quad(n=34)$ de ellos tiene una ansiedad-estado alta, mientras que el 52.1\% $(\mathrm{n}=39)$ tiene una ansiedad-estado media. Respecto a los futbolistas universitarios, se encuentra que el $58.3 \%(n=7)$ tiene una ansiedad-estado media y el restante $41.7 \%(n=5)$ alta; finalmente, de los estudiantes de enseñanza secundaria obligatoria, el 68.0\% ( $n=34)$ tienen una ansiedad-estado media, mientras que el $30.0 \%(n=15)$ tiene ansiedad-estado alta.

Tabla 4. Ansiedad según demarcación y estudios.

\begin{tabular}{|c|c|c|c|c|c|c|}
\hline & & \multicolumn{4}{|c|}{ Demarcación } & \multirow[b]{2}{*}{ Sig } \\
\hline & & $\begin{array}{c}\text { Portero } \\
(\mathrm{n}=27)\end{array}$ & $\begin{array}{c}\text { Defensa } \\
(\mathrm{n}=56)\end{array}$ & $\begin{array}{l}\text { Medio } \\
(\mathrm{n}=35)\end{array}$ & $\begin{array}{c}\text { Delantero } \\
(\mathrm{n}=32)\end{array}$ & \\
\hline \multirow{2}{*}{ Ansiedad-Estado } & Media & $59.3 \%(n=16)$ & $67.9 \%(\mathrm{n}=38)$ & $57.1 \%(n=20)$ & $62.5 \%(n=20)$ & \multirow{2}{*}{.744} \\
\hline & Alta & $40.7 \%(\mathrm{n}=11)$ & $32.1 \%(\mathrm{n}=18)$ & $42.9 \%(\mathrm{n}=15)$ & $37.5 \%(n=12)$ & \\
\hline \multirow{4}{*}{ Ansiedad-Rasgo } & Media & $55.6 \%(\mathrm{n}=15)$ & $42.9 \%(n=24)$ & $48.6 \%(\mathrm{n}=17)$ & $53.1 \%(\mathrm{n}=17)$ & \multirow{2}{*}{.677} \\
\hline & Alta & $44.4 \%(n=12)$ & $57.1 \%(\mathrm{n}=32)$ & $51.4 \%(\mathrm{n}=18)$ & $46.9 \%(\mathrm{n}=15)$ & \\
\hline & & \multicolumn{4}{|c|}{$\begin{array}{l}\text { Estudios } \\
\end{array}$} & \multirow[b]{2}{*}{ Sig } \\
\hline & & $\begin{array}{c}\mathrm{ESO} \\
(\mathrm{n}=49)\end{array}$ & $\begin{array}{c}\text { Bachillerato } \\
(\mathrm{n}=71)\end{array}$ & $\begin{array}{c}\text { Formación Profesional } \\
(\mathrm{n}=18)\end{array}$ & $\begin{array}{c}\text { Universidad } \\
(\mathrm{n}=12)\end{array}$ & \\
\hline \multirow{2}{*}{ Ansiedad-Estado } & Media & $69.4 \%(n=34)$ & $52.1 \%(\mathrm{n}=37)$ & $88.9 \%(\mathrm{n}=16)$ & $58.3 \%(\mathrm{n}=7)$ & \multirow{2}{*}{.021} \\
\hline & Alta & $30.6 \%(n=15)$ & $47.9 \%(n=34)$ & $11.1 \%(\mathrm{n}=2)$ & $41.7 \%(n=5)$ & \\
\hline \multirow{2}{*}{ Ansiedad-Rasgo } & Media & $46.9 \%(\mathrm{n}=23)$ & $49.3 \%(\mathrm{n}=35)$ & $55.6 \%(\mathrm{n}=10)$ & $41.7 \%(\mathrm{n}=5)$ & \multirow{2}{*}{.886} \\
\hline & Alta & $53.1 \%(\mathrm{n}=26)$ & $50.7 \%(n=36)$ & $44.4 \%(\mathrm{n}=8)$ & $58.3 \%(\mathrm{n}=7)$ & \\
\hline
\end{tabular}

En la Tabla 5, en la que se analizan los niveles de ansiedad en función de los años que llevan federados los futbolistas analizados, no se han encontrado diferencias estadísticamente significativas entre la ansiedad-estado y los ańos federado $(p=.929)$, ni entre la ansiedad-rasgo y los ańos federado $(p=.870)$.

$\mathrm{Al}$ analizar las diferencias entre los niveles de ansiedad de los deportistas que conforman el estudio y el trabajo tampoco se ha encontrado asociación estadística $(p=>.05)$.

Tabla 5. Ansiedad según años federado y trabajo.

\begin{tabular}{|c|c|c|c|c|}
\hline & \multicolumn{4}{|c|}{ Años federado } \\
\hline & & $\begin{array}{c}\text { 3-4 ańos } \\
(\mathrm{n}=14)\end{array}$ & $\begin{array}{c}\text { Más de } 5 \text { años } \\
(\mathbf{n}=130)\end{array}$ & Sig. \\
\hline \multirow{2}{*}{ Ansiedad-Estado } & Media & $64.3 \%(n=9)$ & $63.1 \%(\mathrm{n}=82)$ & \multirow{2}{*}{.929} \\
\hline & Alta & $35.7 \%(n=5)$ & $36.9 \%(\mathrm{n}=48)$ & \\
\hline \multirow{2}{*}{ Ansiedad-Rasgo } & Media & $50.0 \%(\mathrm{n}=7)$ & $47.7 \%(n=62)$ & \multirow[b]{2}{*}{.870} \\
\hline & Alta & $50.0 \%(\mathrm{n}=7)$ & $47.7 \%(\mathrm{n}=62)$ & \\
\hline \multicolumn{4}{|c|}{ Trabajo } & \multirow{2}{*}{ Sig. } \\
\hline & & $\mathrm{No}(\mathrm{n}=142)$ & $\mathrm{Si}(\mathrm{n}=8)$ & \\
\hline \multirow{2}{*}{ Ansiedad-Estado } & Media & $63.4 \%(\mathrm{n}=90)$ & $50.0 \%(n=4)$ & \multirow{2}{*}{.446} \\
\hline & Alta & $36.6 \%(\mathrm{n}=52)$ & $50.0 \%(n=4)$ & \\
\hline \multirow{2}{*}{ Ansiedad-Rasgo } & Media & $48.6 \%(\mathrm{n}=69)$ & $50.0 \%(\mathrm{n}=4)$ & \multirow[b]{2}{*}{.938} \\
\hline & Alta & $51.4 \%(\mathrm{n}=73)$ & $50.0 \%(\mathrm{n}=4)$ & \\
\hline
\end{tabular}

\section{Discusión y conclusiones}

Este trabajo de investigación, realizado sobre un total de 156 futbolistas de edades comprendidas entre 16 y 18 años, que competían en las categorías División de Honor, Nacional, Preferente, Primera Provincial y Segunda Provincial, ha contado con porteros, defensas, centrocampistas y delanteros. La mayoría de los futbolistas analizados llevaban más de cinco años practicando este deporte de forma federada y no trabajaban, teniendo un nivel de estudios de formación profesional y bachillerato.

Atendiendo a los niveles de ansiedad-estado de los deportistas analizados, seis de cada diez muestran niveles medios de ansiedad y tres de cada diez tienen una ansiedad alta, y en la ansiedad-rasgo se ha encontrado que la mitad tienen una ansiedad alta y la otra mitad media, cifras normales en jugadores de fútbol, coincidiendo estos resultados con los obtenidos en las investigaciones de Abenza, Olmedilla, Ortega y Esparza (2009) que investigaban la relación entre las lesiones deportivas y diversos factores psicológicos en futbolistas juveniles; la de Olmedilla Álvarez, Ortín y Redondo (2009), en el que se analizaba la ansiedad y la percepción de éxito de los futbolistas, teniendo en cuenta los periodos competitivos a los que se enfrentaban los deportistas y cómo se enfrentaban a ellos; el estudio de Ambrís (2013) realizado sobre futbolistas mexicanos, o el estudio de Fernández et al. (2014), en la que se investigaba sobre las ansiedad en futbolistas, entre otros; sin embargo, estos resultados difie- 
ren de los encontrados por Valdivia-Moral et al. (2016), que encontró que nueve de cada diez judocas analizados tenían niveles de ansiedad alta, cifras muy dispares a las encontradas en esta investigación. Se entiende que las altas cifras de ansiedad media y alta obtenidas en este estudio, aunque sean menores a las obtenidas en investigaciones realizadas sobre deportistas de niveles más altos, se deben a que los futbolistas en los niveles juveniles se preocupan sobremanera por completar las tareas propuestas por los entrenadores y sus cometidos en competición, olvidando que el objetivo principal del deporte en estas etapas es la diversión y el disfrute (Merino, Arraiz y Sabrirón, 2017), aunque no lleguen a los niveles de ansiedad que presentan los deportistas de niveles competitivos más altos.

Los niveles de ansiedad encontrados en los diferentes estudios varían dependiendo de los niveles competitivos en los que se han realizado, encontrando niveles superiores de ansiedad en deportistas semiprofesionales que en deportistas amateur y profesionales, esto se explica por el apoyo del que gozan los atletas de élite, la escasa presión que tienen los deportistas aficionados, y la gran presión y el poco apoyo del que disponen los deportistas semiprofesionales (Prieto, 2017; Zurita, 2015).

Al relacionar los niveles de ansiedad con la categoría en la que compiten los futbolistas analizados no se han encontrado diferencias, datos que difieren de los encontrados por Aguirre-Loaiza y Ramos (2011) en su estudio realizado sobre futbolistas en contextos competitivos, achacando estas cifras a que los deportistas que se encuentran en categorías medias aspirando a conseguir competir en una categoría superior presentan cifras más elevadas de ansiedad que en categorías bajas o en la máxima categoría. Aunque en el presente estudio no se han encontrado estos datos, parece que los niveles de ansiedad aumentan en relación con el número de años que llevan practicando este deporte de forma federada, debido a que al aumentar de categoría la presión por destacar aumenta.

Se ha encontrado que los futbolistas que cursan formación profesional son los que tienen una ansiedad-estado menor, y los que estudian bachillerato son los que muestran niveles de ansiedad más altos. Pero no se han encontrado estudios que contemplen la relación entre estas variables en el fútbol, por lo que no se pueden comparar los datos con otras investigaciones. Probablemente los resultados obtenidos se justifiquen debido a que los jugadores que se encuentran cursando bachillerato tienen menos tiempo libre por la cantidad de tareas, deberes y horas de estudio que necesitan, pudiendo influir esto en el aumento de sus niveles de ansiedad deportiva.
Como principales conclusiones de la presente investigación llevada a cabo en una muestra de 156 futbolistas de edades comprendidas entre 16 y 18 años, que jugaban en las categorías División de Honor, Nacional, Preferente, Primera Provincial y Segunda Provincial, se ha determinado que la mayoría de ellos llevaban más de cinco años jugando al fútbol de forma federada y no trabajaban, siendo su nivel de estudios de Bachillerato y Educación Secundaria Obligatoria en la mayoría.

Respecto a los niveles de ansiedad-estado se ha determinado que seis de cada diez futbolistas analizados muestran niveles medios de ansiedad y tres de cada diez tienen una ansiedad alta. Atendiendo a los niveles de ansiedad-rasgo, se ha encontrado que la mitad tienen una ansiedad alta y la otra mitad media. Los adolescentes que cursan formación profesional son los que tienen una ansiedad-estado menor, y los que estudian bachillerato son los que muestran niveles de ansiedad más altos.

El presente estudio aporta datos importantes sobre los niveles de ansiedad de los futbolistas adolescentes, sirviendo como base para estudios posteriores y para la aplicación de programas de intervención que disminuyan los niveles de ansiedad encontrados en los deportistas, por tratarse de categorías inferiores en las que los futbolistas aún se están formando como deportistas.

Aunque esta investigación no permite establecer recomendaciones para la elaboración de programas de intervención de forma taxativa, por tratarse de un estudio descriptivo de corte transversal con una muestra, parece interesante prestar atención a los niveles de ansiedad de los deportistas de categorías inferiores de fútbol.

Tras comprobar que los niveles de ansiedad de los deportistas son bastante altos, se debe de realizar un seguimiento exhaustivo para controlar estos niveles, aplicando programas de intervención para proporcionarles herramientas psicológicas que ayuden a reducir estos niveles, ya que así aumentará el rendimiento de los mismos. En este caso se deben de llevar a cabo actuaciones basadas en programas que aborden pautas de relajación, como la técnica de Jacobson, el entrenamiento autógeno de Schultz, técnicas de visualización, autohabla positiva o inversión del pensamiento, entre otras. El control de los niveles de ansiedad en los deportistas, independientemente de la categoría competitiva y modalidad deportiva en la que se desenvuelvan, resulta de vital importancia, afectando de forma directa a su rendimiento en competición y desarrollo en situaciones de entrenamiento y mejora, por ello será de vital importancia aplicar las técnicas mencionadas para reducir estos niveles de ansiedad y mejorar la condición de los deportistas. 


\section{Referencias bibliográficas}

1. Abenza, L., Olmedilla, A., Ortega, E., y Esparza, F. (2009). Lesiones y factores psicológicos en futbolistas juveniles. Archivos de medicina del deporte, 132, 280-288.

2. Aguirre-Loaiza, H. H. y Ramos, S. (2011). Ansiedad-estado y variables sociodemográficas en futbolista juveniles colombianos durante competencia. Perspectivas Psicológicas, 7(2), 239-251.

3. Ambrís, J. (2013). Estilos de enseñanza-aprendizaje en relación con la satisfacción y ansiedad que provoca el fútbol en edades tempranas en Ciudad del Carmen (México)(Tesis doctoral). Universidad de Granada, Granada.

4. Campos, G. G., Valdivia-Moral, P., Zagalaz, J. C., Ortega, F. Z., y Romero, O. (2017). Influencia del control del estrés en el rendimiento deportivo: la autoconfianza, la ansiedad y la concentración en deportistas. Retos, 32, 3-6

5. Castro-Sánchez, M., Zurita-Ortega, F., Chacón-Cuberos, R., Martínez-Martínez, A., Espejo-Garcés, T., \& Álvaro-González, J. I. (2015). Harmful substances and motivational climate in relation to physical activity. Health and Addictions/Salud y drogas, 15(2), 115-126.

6. Chacón Cuberos, R., Castro Sánchez, M., Caracuel Cáliz, R., Padial Ruz, R., Collado Fernández, C., \& Zurita Ortega, F. (2016). Profiles of alcohol and tobacco use among adolescents from Andalusia in the first cycle of secondary education. Health and Addictions/Saludy Drogas, 16(2), 93-104.

7. Davids, K., Araújo, D.ySchuttleworth,R.(2005). Applications of Dynamical systems Theoryto Football.En T. Reilly, J. Cabri, D. Araújo (Eds.), Science and Football V (pp.547-560). London: Routledge.

8. Espejo Garcés, T., Martínez Martínez, A., Chacón Cuberos, R., Zurita Ortega, F., Castro Sánchez, M., \& Cachón Zagalaz, J. (2017). Alcohol consumption and physical activity in adolescents from rural environment. Health and Addictions/Saludy Drogas, 17(1), 97-105.

9. Fábregas, A. (2001). Lo sagrado del rebaño. El Fútbol como integrador de identidades. México: El Colegio de Jalisco.

10. Fernández, R., Fidalgo, A.M., Zurita, F., García, J.M. y Sánchez, L. C (2009). Efectos de la hipnosis en la mejora de variables físicas y psicológicas dentro del contexto del deporte. Portales Médicos, 4, 1-5.

11. Fernández, R., Zurita, F., Linares, D., Ambrós, J., Pradas, F., y Linares, M. (2014). Relación entre la ansiedad estado/rasgo, posición en el terreno de juego y ocurrencia de lesiones deportivas. UniversitasPsychologica, 13(2), 433-441.

12. García-Mas, A., Pujals, C., Fuster, P., Núñez, A., y Rubio, V. J. (2014). Determinación de las variables psicológicas y deportivas relevantes a las lesiones deportivas. Revista de psicología del deporte, 23(2), 0423-429.

13. González-Campos, G. (2016). Estilos de enseńanza de entrenadores y su relación con la ansiedad de los jugadores en diferentes categorías de futbol base. Sportis: Revista Técnico-Cientifica del Deporte Escolar, Educación Física y Psicomotricidad, 2(3), 390-411.

14. Johnson, U., eIvarsson, A. (2010). Psychologicalpredictors of injuries among junior soccer players.ScandinavianJournal of Medicine and Science in Sport, 21(1), 129-136.

15. Leal, F. M. (2016). Estudio de las variables que participan en los estilos de liderazgo de entrenadores deportivos de baloncesto desde el modelo multidimensional. Liminales. Escritossobrepsicología y sociedad, (5), 71-94.

16. León-Prados, J. A., Fuentes, I., y Calvo, A. (2016). Relación entre ansiedad estado, autoconfianza percibida y rendimiento en baloncesto/ Relationshipbetweenanxietystate, self-confidence and performance in basketball. Revista Internacional de Medicina y Ciencias de la Actividad Física y del Deporte, 55(1), 1-12.

17. Liberal, R., Escudero, J. T., Cantallops, J., y Ponseti, X. (2014). Impacto psicológico de las lesiones deportivas en relación al bienestar psicológico y la ansiedad asociada a deportes de competición. Revista de psicología del deporte, 23(2), 451-456.
18. Loerhr, J. (1990). La excelencia en los deportes. México: Planeta.

19. McAuley, E., Márquez, D.X., Jerome, G.J., Blissmer, B., y Katula, J. (2002). Physical activity and psique anxiety in older adults: fitness, and efficacy influences. Aging and Mental Health, 6, 222-230.

20. Merino, A., Arraiz, A. y Sabirón, F. (2017). La adherenciadelentorno familiar en el fútbolprebenjamín: un estudio de caso. Revista iberoamericana de psicología del ejercicio y el deporte, 12(1), 139-148.

21. Molina, J., Sandín, B., y Chorot, P. (2014). Sensibilidad a la ansiedad y presión psicológica: Efectos sobre el rendimiento deportivo en adolescentes. Cuadernos de psicologia del deporte, 14(1), $45-54$.

22. Mora, À., Sousa, C., y Cruz, J. (2014). El clima motivacional, la autoestima y la ansiedad en jugadores jóvenes de un club de baloncesto. Apunts. Educació fisica i esports, 17(1), 43-51.

23. Morales, A., y Guzmán, M. (2000). Diccionario Temático de los Deportes. Málaga: Arguval.

24. Moreno-Pellicer, R. (2017). Estudio de variables motivacionales y de liderazgo y su relación con el bienestar y malestar en fútbol categoría juvenil en la comarca de la Safor(Tesis doctoral). Universidad de Valencia, Valencia.

25. Olmedilla, A., Álvarez, A., Ortín, F. J., y Redondo, A. (2009). Ansiedad competitiva, percepción de éxito y lesiones: un estudio en futbolistas. Revista Internacional de Medicina y Ciencias de la Actividad Física y del Deporte, 9(33), 14-26.

26. Olmedilla, A., Bazaco, M.J., Ortega, E. y Boladeras, A. (2011). Formación psicológica en futbolistas juveniles con el bienestar psicológico percibido, la ansiedad y la satisfacción. Revista Electrónica de Psicología, 12, 221-237

27. Olmedilla, A., Prieto, J. G. y Blas, A. (2011). Relaciones entre estrés psicosocial y lesiones deportivas en tenistas. Universitas Psychologica, 10(3), 909-922.

28. Pazo, C. I., Sáenz-López, P. y Fradua, L. (2012). Influencia del contexto deportivo en la formación de los futbolistas de la selección española de fútbol. Revista de Psicología del Deporte, 21(2), 291-299.

29. Prieto, J. (2017). Relación entre competitividad, ansiedad social y compromiso con variables deportivas y académicas en futbolistas jóvenes. Revista iberoamericana de psicología del ejercicio y el deporte, 11(2), $193-$ 200.

30. Rosenthal,M.D., Rainey, C.E., Tognoni, A. y Worms, R. (2012).Evaluation and management of posterior cruciate ligament injuriesPhysical therapy, 13(4), 196-208.

31. Smith, R.E., Smoll, F.L. y Cumming, S.P. (2007). Effects of a motivational climate intervention for coaches on young athletes' sport performance anxiety. Journal of Sport Psychology, 1, 59-75.

32. Spielberg, C., Gorsuch, R. L., y Lushene, R. E. (1970). The state-trait anxiety inventory. Palo Alto, CA: Consulting Psychologists Press.

33. Spielberger, C. D., Gorsuch, R. L., Lushene, R., Vagg, P. R. y Jacobs, G. A. (1983). Manual for the state-trait anxiety inventory, (Form Y). Palo Alto. CA: Consulting Psychologists Press.

34. Valdivia-Moral, P. (2016). Niveles de ansiedad, autoconcepto y resiliencia en deportes individuales(Tesis doctoral). Universidad de Granada, Granada.

35. Valdivia-Moral, P., Zafra, E., Zurita, F., Castro-Sánchez, M., Muros, J. J., y Cofre-Bolados, C. (2016). Niveles de Ansiedad en Judocas chilenos. Journal of Sport and Health Research, 8(2), 129-138.

36. Weinberg, R.S. y Gould, D. (1996) Fundamentos de psicología del deporte y del ejercicio físico. Barcelona: Ariel.

37. Weinberg, R.S. y Gould, D. (2010). Fundamentos de Psicología del Deporte y del Ejercicio físico. Madrid: Panamericana.

38. Yuill, E., Pajackkowsky, J., Jason, A. y Howitt, S. (2012). Conservative 
care of sport hernias within soccer players: A case series. Journal of Bodywork and Movement Therapies, 16(4), 540-548.

39. Zafra-Santos, E. O. (2016). Capacidad de resiliencia frente a lesiones deportivas y su relación con el desempeño deportivo en judocas de Chile(Tesis doctoral). Universidad de Granada, Granada.

40. Zurita, F. (2015). Influencia de los factores psicológicos sobre las lesiones deportivas en deportes de equipo(Tesis doctoral). Universidad de Granada, Granada.

41. Zurita, F., Pérez, A. J., González, G., Castro, M., Chacón, R., y Ambrís, J. (2016). Estilos de enseñanza-entrenamiento y relación con ansiedad de jugadores de iniciación al futbol. Sportis, 2(3), 390-411. 